\title{
A new method to study inhomogeneities in climate records: \\ Brownian Motion or Random Deviations?
}

by

Ralf Lindau and Victor Venema

Meteorological Institute

University of Bonn

Auf dem Hügel 20

D 53121 Bonn

Germany

Correspondence to Ralf Lindau (rlindau@uni-bonn.de)

This is a preprint. This manuscript was

published after minor revisions in the

International Journal of Climatology.

Article DOI: 10.1002/joc.6105

Short title: Brownian motion or Random deviations?

Keywords: Homogenization, climate stations, Brownian motion, Random Deviations, signal separation, variance of lag differences, number of breaks, spurious trends 
Abstract. Climate data is affected by inhomogeneities due to historical changes in the way the measurements were performed. Understanding these inhomogeneities is important for accurate estimates of long-term changes in the climate. These inhomogeneities are typically characterized by the number of breaks and the size of the jumps or the variance of the break signal, but a full characterization of the break signal also includes its temporal behavior. This study develops a method to distinguish between two types of breaks: random deviations from a baseline and Brownian motion. Strength and frequency of both break types are estimated by using the variance of the spatiotemporal differences in the time series of two nearby stations as input. Thus, the result is directly obtained from the data without running a homogenization algorithm to estimate the break signal from the data. This opens the possibility to determine the total number of breaks and not only that of the significantly large ones. The application to German temperature observations suggests generally small inhomogeneities dominated by random deviations from a baseline. US stations, on the other hand, show also the characteristics of a strong Brownian motion type component.

\section{Introduction}

Climate station records are affected by inhomogeneities resulting from relocations of stations or changes in the measuring methods. To study long-term changes in the climate accurately the influence of these inhomogeneities on observational data needs to be reduced as much as possible. How well this is possible using statistical homogenization algorithms depends on the statistical properties of the inhomogeneities and of the station network.

Inhomogeneities are detected and corrected by comparing neighboring stations with each other. For temperature data these comparisons are performed by computing a difference time series of a candidate station and its neighboring reference station. This removes the complicated and noisy regional climate signal and makes it much easier to detect and adjust the inhomogeneities.

The most important properties of the inhomogeneities are their size and frequency. However, these properties do not fully describe inhomogeneities present in a station series; also important is the temporal behavior of the inhomogeneities. In this article we will study two classical types of processes with very different temporal behaviors: Random Deviations from a baseline (RD) and Brownian Motion (BM). As explained in Section 2 in more detail, this matters because for the same break sizes these two behaviors differ in the total variance of the break signal and in how large the trend errors due to the inhomogeneities are.

The realism of benchmark datasets is important for a fair comparison of homogenization algorithms and vital for accurate estimates of errors remaining in the data after homogenization. Early validation datasets often had only one break inhomogeneity or only large ones, while not allowing breaks to be close together. They also often did not have an explicit reference series or assumed the reference was homogeneous.

Menne and Williams (2005) generated an annual dataset with randomly positioned breaks in the reference time series and simulated the break signal as Brownian Motion, just as Menne and Williams (2009) and Williams et al. (2012). Furthermore, Menne and Williams (2005) studied the sizes of breaks known from station histories and found that break sizes have a normal distribution, including small breaks. We will use this distribution to model breaks in this paper. 
Domonkos (2011) generated several validation datasets from simple to complex. In the complex socalled Hungarian standard dataset the inhomogeneities were tuned to make the properties of the detected breaks fit to those of the detected breaks in the Hungarian network. The breaks were in principle simulated as Brownian Motion, but the maximum deviation was limited (Domonkos, pers. Comm., 2018). Furthermore, also many platform-like break pairs were inserted. The latter pairs with opposite signs make the sign statistics of consecutive breaks more similar to Random Deviations, but the long-term behavior would still be similar to Brownian Motion.

The above mentioned papers do not explicitly mention that they model breaks as Brownian Motion. By accident the simulated data of the benchmarking study of the COST Action HOME (2007-2012) used Random Deviations (Venema et al., 2012). Later studies used both processes. A study on daily temperature have generated data that is mostly Brownian Motion (Killick, 2016), while newer studies on monthly temperature and daily humidity inserted breaks as Random Deviations (Guijarro et al., 2017; Chimani et al. 2018).

In case of Brownian Motion the probability of a break going up or down is always $50 \%$, independent of the previous level or jump size. However, for Random Deviation from a baseline the statistic is different: 2 of 3 break pairs will have opposite signs (platform-like break pairs). Analyzing the sign statistics of consecutive detected breaks Venema et al. (2012) found that pairs with opposite signs were well above $50 \%$ (not fitting to pure Brownian Motion). However, this percentage was slightly lower in the real European networks (59\%) than in the simulated Random Deviation data (63\%) . These statistics suggest that breaks mostly behave like Random Deviations, but also provide weak evidence that there may be a Brownian Motion component.

The article is structured as follows. In the next section we explain why it matters whether the break signal is modelled as Random Deviations or Brownian Motion. For the same break sizes the variance of the break time series and the trend errors due to the breaks are much larger for Brownian Motion. In Section 3 we describe our statistical model for the break signals and introduce the variance of the spatiotemporal difference considered as a function of time lag. This function is used as a metric that allows us to decompose a break signal into its RD and BM components. We derive equations how this metric depends on the strength of the noise as well as on the number and size of breaks for RD and BM inhomogeneities. In Section 4 we test this method by simulated data and propose a retrieval method for the characteristic parameters of the break signals. For accurate estimates of these parameters the method needs a large number of stations.

The method is applied to monthly temperature observations from the USA and Germany in Section 5. In Germany the break signal is dominated by Random Deviations, no sign of Brownian Motion is found. In the USA there is a Brownian Motion component and the total number of estimated breaks is found to be large. For this reason we study in Section 6 the fraction of breaks that can be detected by statistical homogenization, which can explain a large part of the difference between the number of detected breaks reported in the literature and the number our retrieval finds. The article finishes with Discussions (Section 7) and Conclusions (Section 8).

\section{The different characteristics of Brownian motion and Random deviation}

Figure 1 shows a conceptual model how to describe an identical break signal by two different approaches. In both cases a random variable $\Delta T$ produces a typical break signal in form of a step 
function. The question is which of the two sketched $\Delta T$ is assumed to be an independent random variable, the heights of the segment levels itself or the jumps heights between two levels? In the first case Random deviations (RD) result (Fig. 1a), in the second a Brownian motion (BM) (Fig. 1b). Depending on our choice different statistical properties of the break signal will result.

The variance of an RD process is in principle equal to $\sigma_{\delta}{ }^{2}$, the variance of the random numbers used to create the segment levels. In this estimation we neglect the slightly increased effective variance due to the assumed different lengths of the segments, which are caused by the stochastic occurrence of the breaks (Lindau and Venema, 2018b). Nevertheless, the jump heights between two levels consist of the sum of two of such independent random numbers so that their variance $\sigma_{\beta}{ }^{2}$ is twice as large.

$$
\operatorname{Var}_{R D}=\sigma_{\delta}^{2}=\frac{\sigma_{\beta}^{2}}{2}
$$

The variance of a BM process grows linearly in time, because the $k^{\text {th }}$ value is defined as the sum of $k$ random independent numbers. Thus, at the end of a BM time series the variance is equal to $k \sigma_{\beta}^{2}$ where $k$ denotes the number of breaks and $\sigma_{\beta}{ }^{2}$ the jump height variance. Since the variance is linearly growing in time, the average variance over time is equal to the half:

$$
\operatorname{Var}_{B M}=k \frac{\sigma_{\beta}^{2}}{2}
$$

A comparison of Eq. (1) and (2) shows that the variance of a BM-break signal is $k$ times larger compared to an RD-break signal with the same magnitude of jump sizes, which is often used to characterize the break signal. The latter could also explain why many authors actually assume BM type breaks. If the jump heights are used to characterize the break sizes, it feels appropriate to assume that they can be modelled as an independent random variable, which leads automatically to BM type breaks.

Following Venema et al. (2012) we usually assume five breaks within a time series of 100 years for a typical European station. A difference time series will have twice as much breaks as two stations contribute. Thus, BM type breaks produce a 10 times larger variance in the difference time series compared those of random deviation type, when the jump sizes are equally large.

With a ten times larger variance, BM type breaks should be visible already by eye and the linearly growing variance seems unrealistic especially for longer time series. But the above discussion deals with the total variance. Differences in the mean of candidate and reference contribute to this total variance. However, this difference is partially due to real differences of the climate normals between two stations of unknown magnitude. As a consequence we are forced to remove this mean difference.

Following Lindau (2003) and Lindau and Venema (2013), the total variance can be decomposed into an external and an internal part. This rule is often referred as to Law of the Total Variance:

$$
\operatorname{Var}(X)=\operatorname{Var}(\mathrm{E}[X])+\mathrm{E}[\operatorname{Var}(X)]
$$

Eq. (3) states that the variance of the external averages plus the mean internal variance constitutes the total variance. In the present case we consider several time series of a dataset and apply the 
above variance decomposition. The variance between the different means of each time series is considered as external and the mean variance within the times series as internal. As shown in the Appendix, two thirds of the total variance of a BM process is allotted to the variance of the means (the external part) and only one third to the internal variance. Because real climatic differences between two stations also contribute to the external variance we need to remove it completely in our data analysis and thus inherently also remove that part of the external variance, which is caused by the inhomogeneities. The remaining internal variance amounts to only $\sigma_{\beta}{ }^{2} k / 6$. For RD breaks the major part of the variance (a fraction of $(k-1) / k$ ) is internal. Thus, for $k=6$ the internal $B M$ variance is about twice as large as for RD. However, the break variance by itself cannot be used to distinguish the cases. The more so as observed data can have both BM and RD characteristics.

Even more important are the different effects the two breaks types will have on the trend. Lindau and Venema (2018b) investigated the trend introduced by RD breaks. Assuming zero mean jump sizes they found that the error variance of the trend decreases with the number of breaks. This can be understood considering that more underlying independents will make the random fluctuations of the trend smaller.

BM breaks have the opposite effect. To estimate the trend error consider that the last homogeneous subperiod (HSP) of a Brownian motion has the expected value zero and varies with a standard deviation of $\sqrt{k} \sigma_{\beta}$, whereas the first one is constant and zero. The difference between the first and last HSP divided by the length $n$ does not give the completely correct trend, but is nevertheless a good estimate of its magnitude. Thus, for BM the error variance of the trend grows linearly with $k$, the number of breaks.

For a long time we were not fully aware that there are two different approaches. Williams et al. (2012) modelled BM type breaks whereas Venema et al. (2012) inserted RD type breaks into their benchmark dataset. Only the standard deviations applied to generate the break sizes were communicated. These should be reduced by a factor of $\sqrt{2}$ when transferred from BM to RD, but, most important, the statistical properties of the signals differ considerably depending on the break type created.

As mentioned in the introduction, Venema et al. (2012) briefly raised this topic and analyzed the statistics of the break signal to decide whether the detected breaks are of BM or RD type. An important difference to the present study is that their results are based on the retrieved break signal, which may differ from the true one. We assume the break signal to be a step function that consists of a number of homogeneous subperiods (HSPs). Venema et al. (2012) considered triples of consecutive HSPs and distinguished between stairs and platforms. Stairs are defined by a monotonic increase or decrease, whereas for platforms the middle HSP is either the maximum or the minimum of the considered triple. The idea is that the percentage of stairs and platforms should be equal for BM because the second break is independent of the first break, whereas platforms should occur more frequently in RD data, because there is a tendency to revert back to the baseline. The considered triples are defined by three random numbers (the levels of the three HSPs). There are six different possibilities of rank order for these three values, which for RD all have the same probability. One combination is an upward stair, a second one is a downward stair, and the other four possibilities are platforms. Consequently, the theoretical fraction of platforms for RD breaks is $2 / 3$. 
As mentioned above, Venema et al. (2012) found only 62 to $64 \%$ platforms for the benchmark data containing pure artificial RD type breaks, although a fraction of $2 / 3$ is expected. This suggests that stairs are easier to detect than platforms, although also gradual inhomogeneities modelled as linear trends in the station data could have lowered the percentage of detected platforms. The percentage of stairs and platforms as they are detectable by a homogenization algorithm will be further dependent, e.g., on the signal-to-noise ratio (SNR), which is shown to be a key parameter for break detection (Lindau and Venema, 2016; Lindau and Venema, 2018a). Thus, to conclude that the reduced number of detected platforms is directly caused by an admixture of BM-type breaks is risky and has been a motivation for this further study.

\section{Methodology}

\subsection{Stochastic simulation}

To analyze the effect of the different break types, we used simulated data that is described in the following. We generated time series with a total length of $n$ years:

$$
x(i), i=1,2,3, \ldots, n
$$

that consists of four superimposed signals:

1. The climate signal $\gamma$, which is identical for all stations of a network

2. Noise $\varepsilon$ added to the climate signal, which mimics the differences between the stations within a network, e.g., due to the weather

3. BM-type inhomogeneities $\beta$

4. RD-type inhomogeneities $\delta$

$$
x(i)=\gamma(i)+\varepsilon(i)+\beta(i)+\delta(i)
$$

We assume that the BM and RD-type break signals are independent from each other. Further, the timings of the breaks occur randomly with a mean frequency, which may be different for the two types. For both break types the signal remains at a constant level between two adjacent break points. For RD-type breaks these levels are random, independent and normal distributed. For the BM-type inhomogeneities, this is not the case for the levels itself, but for the jumps from one level to the next one.

\subsection{Spatiotemporal differences}

Our approach distinguishes the two break signals by analyzing the variance of the spatiotemporal differences of the data. First, the spatial difference time series dif between two neighboring stations $x_{1}$ and $x_{2}$ is built, which eliminates the common climate signal $\gamma$.

$$
\operatorname{dif}(i)=x_{1}(i)-x_{2}(i)=\left(\beta_{1}(i)+\delta_{1}(i)+\varepsilon_{1}(i)\right)-\left(\beta_{2}(i)+\delta_{2}(i)+\varepsilon_{2}(i)\right)
$$

Then, temporal differences $D$ (with $\operatorname{lag} L$ ) within the time series dif are built:

$$
D=\operatorname{dif}(i)-\operatorname{dif}(i+L)
$$


Inserting Eq. (6) into Eq. (7), we get:

$$
\begin{gathered}
D=\left(\beta_{1}(i)+\delta_{1}(i)+\varepsilon_{1}(i)\right)-\left(\beta_{2}(i)+\delta_{2}(i)+\varepsilon_{2}(i)\right)-\left(\beta_{1}(i+L)+\delta_{1}(i+L)+\varepsilon_{1}(i+L)\right) \\
+\left(\beta_{2}(i+L)+\delta_{2}(i+L)+\varepsilon_{2}(i+L)\right)
\end{gathered}
$$

Thus, $D$ consists of the sum (or difference) of twelve numbers with known statistical properties: three types $\left(\beta, \delta\right.$, and $\varepsilon$ ) from two stations $\left(x_{1}\right.$ and $\left.x_{2}\right)$ and two time points $(i$ and $i+L)$. In a final step, the variance of $D$ is calculated for classes of constant time lags $L$.

It is a general rule that the variance of the sum (or the difference) of two variables is equal to the sum of their variances increased (or reduced) by twice the covariance between them:

$$
\operatorname{Var}(a \pm b)=\operatorname{Var}(a)+\operatorname{Var}(b) \pm 2 \operatorname{Cov}(a, b)
$$

Applying Eq. (9) to Eq. (8), we obtain:

$$
\begin{gathered}
\operatorname{Var}(D)=2 \operatorname{Var}(\beta(i))+2 \operatorname{Var}(\beta(i+L))+4 \operatorname{Var}(\delta)+4 \operatorname{Var}(\varepsilon)-4 \operatorname{Cov}(\beta(i), \beta(i+L)) \\
-4 \operatorname{Cov}(\delta(i), \delta(i+L))
\end{gathered}
$$

In Eq. (10), twelve variance terms occur, one for each for the twelve variables in Eq. (8). The four terms for RD-type breaks does not depend on time so that they can be summarized to one term only. The same is true for the noise variance. However, for BM-type inhomogeneities the variance grows with time so that two separate terms, one for time step $i$ and another for time step $i+L$, must be used. Most of the covariances between the twelve variables are zero by definition so that they can be omitted. This is trivial for the noise, but also valid for the inhomogeneities when different stations or types are considered. Only the temporal covariance for identical stations and types are non-zero. These occur two times for each station and a further factor 2 comes directly from the definition in Eq. (9). Together, they lead to the factor 4 in front of the covariance terms.

\subsection{Random Deviations}

The last term in Eq. (10) denotes the covariance for RD-type breaks, where the years $i$ and $i+L$ are considered. For this break type, the levels of two different inhomogeneity segments are independent. Only for internal pairs, i.e. if $i$ and $i+L$ are both situated in the same segment, the covariance is equal to the variance $\operatorname{Var}(\delta)$ itself; in all other cases it is zero. Consequently, the covariance is equal to the variance multiplied by the probability of internal pairs $P_{\text {int }}$.

$$
\operatorname{Cov}(\delta(i), \delta(i+L))=P_{\text {int }} \operatorname{Var}(\delta)
$$

As mentioned above, we assume that breaks occur with a mean frequency $p_{\delta}$. Consequently, the probability $f$ to find $k$ breaks within a time span $L$ is Poisson-distributed:

$$
f(k)=\frac{\lambda^{k} e^{-\lambda}}{k !} \quad, \quad \text { with } \lambda=p_{\delta} L
$$

Setting $k=0$ in Eq. (12) yields an equation for the probability of internal pairs:

$$
P_{\text {int }}=f(0)=e^{-\lambda}=e^{-p_{\delta} L}
$$

By inserting Eq. (13) into Eq. (11) we finally get: 


$$
\operatorname{Cov}(\delta(i), \delta(i+L))=\operatorname{Var}(\delta) e^{-p_{\delta} L}
$$

\subsection{Brownian Motion}

Now we consider the three $\beta$-terms in Eq. (10), which describe the BM-type breaks. A classical Brownian motion is defined as following:

$$
\beta(i)=\sum_{j=1}^{i} a(j), \quad \beta(0)=0, \quad a \sim N\left(0, \sigma_{\beta}{ }^{2}\right)
$$

with $a$ being a random variable. The variance of this process increases linearly in time, because at each time step a growing sum of random, independent and normal distributed numbers is obtained. The variance at time step $i$ is:

$$
\operatorname{Var}(\beta(i))_{\text {classic }}=i \sigma_{\beta}^{2}
$$

However, in our case, a new value is created not every year, but only in years when actually a break occurs, which happens only with a probability of $p_{\beta}$. Consequently, the variance growth is reduced by this factor.

$$
\operatorname{Var}(\beta(i))=i p_{\beta} \sigma_{\beta}^{2}
$$

and analogously:

$$
\operatorname{Var}(\beta(i+L))=(i+L) p_{\beta} \sigma_{\beta}^{2}
$$

The covariance of two time steps within a Brownian motion is equal to the variance of the earlier one, because both values have all random numbers in common that produce the earlier value. Any additional break after the first time step do not contribute to the covariance.

$$
\operatorname{Cov}(\beta(i), \beta(i+L))=i p_{\beta} \sigma_{\beta}^{2}
$$

Summarizing Eq. (17a), (17b), and (18) yields:

$$
\operatorname{Var}(\beta(i))+\operatorname{Var}(\beta(i+L))-2 \operatorname{Cov}(\beta(i), \beta(i+L))=L p_{\beta} \sigma_{\beta}^{2}
$$

\subsection{The overall variance of spatiotemporal differences}

We insert Eqs. (14) and (19) into Eq. (10) and replace the expressions $\operatorname{Var}(\delta)$ and $\operatorname{Var}(\varepsilon)$ by $\sigma_{\delta}{ }^{2}$ and $\sigma_{\varepsilon}^{2}$, respectively:

$$
\operatorname{Var}(D(L))=2 p_{\beta} \sigma_{\beta}^{2} L+4 \sigma_{\delta}^{2}\left(1-e^{-p_{\delta} L}\right)+{\sigma_{\varepsilon}}^{2}
$$

Eq. (20) shows that we are able to identify the different effects of BM-type breaks, RD-type breaks, and noise by calculating the variance of spatiotemporal differences of climate time series as described in this section. This is possible since the three processes produce different types of signals: The first term on the right-hand side describes the signal of BM-type breaks, which grows linearly 
with $L$; the second term denotes RD-type breaks, which can be identified by a saturating exponential growth of the variance; the third term, the noise, produces a constant offset.

\section{Application to simulated data}

In this section the developed method is applied to simulated data. We generated 1000 pairs of time series with a length of $n=100$ as described in Section 3. In a first step the two different break types are inserted separately. Fig. 2a shows the result for RD-type breaks alone. In this example we set the noise variance $\sigma_{\varepsilon}^{2}=0.05 \mathrm{~K}^{2}$, the RD break variance $\sigma_{\delta}^{2}=0.10 \mathrm{~K}^{2}$, and the frequency of breaks $p_{\delta}=$ $0.05 \mathrm{a}^{-1}$. The O-symbols give the empirically obtained variance $V_{\text {emp }}$ for each time lag $L$, whereas the fat line denotes the theoretical expectation of $V$ according to Eq. (20). Data and theory are in almost perfect agreement, both showing a saturating exponential increase (by $1-\mathrm{e}^{-\mathrm{x}}$ ) with a saturation level for large $L$ and a constant offset caused by the noise. When only BM breaks plus noise (with $\sigma_{\beta}{ }^{2}=$ $0.05 \mathrm{~K}^{2}$ and $p_{\beta}=0.05 \mathrm{a}^{-1}$ ) and no RD breaks are inserted (Fig. 2b), the variance increases linearly as predicted by the theory. Fig. 2c shows the result for a mixture of both break types with a non-linear steep increase of variance for small lags and a transition to a linear increase, which is slower because at larger lags only BM breaks contribute. Again, the simulated data is closely following the theory, which shows that the method suggested in Section 3 works.

\subsection{Retrieval for the characteristic parameters}

In Fig. 2 the known five parameters used to generate the data (i.e., strength of noise and strength and frequency of both break types) are merely inserted into Eq. (20) to show the good agreement between simulated data and theory. However, for real observations these parameters will not be known a priori, but they have to be retrieved by fitting a function of the form given in Eq. (20) to the spatiotemporal variance data. In the following we describe the fitting procedure and investigate how accurate the application is.

Eq. (20) can be concisely written as:

$$
V(L)=b L+d\left(1-e^{-c L}\right)+e
$$

$V(L)$ has four free parameters. A comparison with Eq. (20) shows:

$$
\begin{aligned}
& b=2 p_{\beta} \sigma_{\beta}^{2} \\
& c=p_{\delta} \\
& d=4{\sigma_{\delta}}^{2} \\
& e=4{\sigma_{\varepsilon}}^{2}
\end{aligned}
$$

This is one parameter less (4) than necessary to generate the data (5). The strength and frequency of the BM type breaks cannot be separated from each other, but arise as product in parameter $b$; many small BM-type breaks have the same effect as a few large one.

We fit the function given in Eq. (21) by a two-step approach. Initially, a first guess of the four parameters is computed, which is then used as central point of an exhausting search in the 
surrounding. The first guess is obtained by fitting two tangents, one for $L \rightarrow 0$ and the other for $L \rightarrow \infty$. Technically, the two tangents $V_{1}$ and $V_{2}$ are derived by fitting a straight line to the data curve between $L=1$ to 3 and $L=51$ to 80 , respectively.

For $L \rightarrow 0$, we use $e^{-x} \approx 1-x$ so that Eq. (21a) can be approximated by:

$$
V_{1}(L)=b L+c d L+e=\operatorname{slp}_{1} L+\operatorname{con}_{1}
$$

with:

$$
\begin{aligned}
& \operatorname{slp}_{1}=b+c d \\
& \operatorname{con}_{1}=e
\end{aligned}
$$

For $L \rightarrow \infty$, the exponential term in Eq. (21a) can be neglected and it follows:

$$
V_{2}(L)=b L+d+e=\operatorname{slp}_{2} L+\operatorname{con}_{2}
$$

with:

$$
\begin{gathered}
\operatorname{slp}_{2}=b \\
\operatorname{con}_{2}=d+e
\end{gathered}
$$

Eq. $(22 \mathrm{~b} / \mathrm{c})$ and $(23 \mathrm{~b} / \mathrm{c})$ give four equations for the four characteristic parameters yielding the first guess, which we mark by a zero index:

$$
\begin{aligned}
& b_{0}=\operatorname{slp}_{2} \\
& c_{0}=\frac{s l p_{1}-s p_{2}}{\operatorname{con}_{2}-\operatorname{con}_{1}} \\
& d_{0}=\operatorname{con}_{2}-\operatorname{con}_{1} \\
& e_{0}=\operatorname{con}_{1}
\end{aligned}
$$

The final fit is performed by varying each of the four first guess parameters in 41 samples around the initial value and testing which of the $41^{4}$ combinations yields a minimum in the squared difference $\Delta$

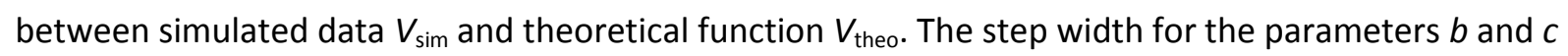
is 0.001 and 0.01 for $d$ and $e$. The number of data pairs decrease linearly with $L$, which is taken into account by an according weighting factor.

$$
\Delta(b, c, d, e)=\sum_{L=1}^{L_{\max }}\left(L_{\max }-L\right)\left(V_{\text {sim }}(L)-V_{\text {theo }}(L)\right)^{2}
$$

Fig. 3 illustrates the above described parameter retrieval. Using the five input parameters (given in the upper left corner), 10,000 time series pairs are generated. Their mean spatiotemporal variance for each time lag is denoted by $O$-symbols. The obtained retrieval is given by a curve that is in nearly perfect agreement with the data. In the lower right corner the retrieved parameters $b, c, d$, and $e$ defining this curve are explicitly shown. Further, the two tangents $V_{1}$ and $V_{2}$ are shown, which offer the possibility to interpret the found parameters geometrically by using Eq. (24). 
The parameters $d$ and $e$ have the physical dimension $\mathrm{K}^{2}$ and describe the strength of RD type breaks and noise, respectively. They can be read at the $y$-axis: $e$ is equal to the intercept of the tangent $V_{1}$, whereas the intercept of $V_{2}$ is giving the sum of $d$ and $e$. For both parameters the expected value is $0.4 \mathrm{~K}^{2}$, which can be obtained by inserting the according input values into Eq. (21d) and (21e). With 0.38 and $0.39 \mathrm{~K}^{2}$ actual estimates differ by a few percent.

For the characterization of BM type breaks we have only one parameter $b$ combining strength and frequency. It has the dimension $\mathrm{K}^{2} \mathrm{a}^{-1}$ and is represented by the slope of tangent $V_{2}$. The retrieval yields $0.0105 \mathrm{~K}^{2} \mathrm{a}^{-1}$, which differs by $5 \%$ from the expectation $(0.0100)$ given in Eq. (21b).

The parameter $c$ denotes the frequency of RD type breaks, which has the dimension $\mathrm{a}^{-1}$. Its reciprocal, the average time between two break events, can be read as the x-value of the crossing point of the two tangents as indicated by the vertical dashed line in Fig. 3. Its expectation is matched better than $1 \%$ ( 0.0500 vs. 0.0498$)$.

So far we considered the result of 10,000 data pairs to show that the method works in principle. However, for applications to real observations we may have considerably less data, which will diminish the accuracy of the retrieval. To estimate this effect, we reduced the number of pairs drastically to 100 and repeated the procedure five times to get an idea how much the results vary. Fig. 4 shows the outcome separately for the five randomly different datasets (again given by 0 symbols) together with the fitted curve (given by the solid lines). The spread between the curves is large especially for higher lags. The problem is mainly that the data itself varies between the different samples, whereas the retrieved curve fits well to the according data. These random fluctuations are quantified by the standard deviation for each parameter in Fig. 4 . For the parameters $b, c$, and $d$, they reach about $50 \%$ when expressed as relative error in the mean. The error of the noise parameter $e$ is, with about 5\%, much smaller. Increasing the number of repetition from 5 to 20 , the results are more stable and summarized in Table 1; Table 2 shows the according findings for 1000 data pairs.

The accuracy increases when only RD breaks and noise are inserted, but no BM breaks. For 100 station pairs the standard deviation of the four parameters $b, c, d$, and $e$ decreases to 0.133 (from 0.317 ), 1.812 (from 2.618), 0.098 (from 0.184), and 0.009 (from 0.015). The numbers in brackets are extracted from Tab. 1 for comparison.

\section{Application to observations}

Finally, the above described method is applied to real observations. We used data of the International Surface Temperature Initiative (ISTI) (Rennie et al., 2014). In a first step we restricted the data to the $20^{\text {th }}$ century to limit effects of possible non-stationarities. Spatial difference time series are built against the best neighbor station, which is defined as the nearest neighbor within a maximum distance of $100 \mathrm{~km}$ and with at least 80 years of overlapping data. To avoid mutual double pairs, the best neighbor is only searched in eastward direction. In the contiguous United States, 1459 station pairs fulfil these constraints (Fig. 5). Temporal differences are built from monthly resolution data, but only between identical calendar months to avoid any spurious effect caused by the annual cycle. Thus, only whole year lags are possible, but the resulting parameters are valid for monthly resolution. 
The resulting variance of the spatiotemporal difference according to Eq. (20) is given in the left panel of Fig. 5. For time lags smaller than 20 years the saturating exponential increase induced by RD type breaks is obvious; for larger time lags the increase is clearly linear indicating that also BM type breaks are included in the data.

For Germany only 45 station pairs (Fig. 6) could be built, which is certainly at the lower limit for a useful application of the method. However, compared to US, German data shows a distinctly different behavior. Especially, any evidence for BM-type breaks is missing, as the curve saturates at a constant level for higher time lags. Reconverting the coefficients $b$ to $e$ by Eq. (21b-e) we get the estimates summarized in Table 3.

The standard deviations for noise and RD-type breaks are of comparable size with 0.3 to $0.4 \mathrm{~K}$ for both Germany and US. However, there are four times more RD type breaks in US data (one each 6 years compared to one each 24 years).

The break sizes are commonly communicated as the standard deviation of the jump heights between two adjacent HSPs. To convert the variance $\sigma_{\delta}^{2}$ given in Table 3 into this traditional parameter, we have to multiply by 2 (as two RDs build one jump) and calculate the square root. For US data $0.485 \mathrm{~K}$ results, for Germany $0.505 \mathrm{~K}$.

In Section 2 we roughly estimated the error variance of the temperature change caused by BM breaks to $k \sigma_{\beta}{ }^{2}$. This is equal to the BM parameter $p_{\beta} \sigma_{\beta}{ }^{2}$ (Table 3) multiplied by the length of the series, which gives for US stations $0.45 \mathrm{~K}^{2}$ for a length of 100 years. Thus, the BM-type breaks cause an error of about $0.67 \mathrm{~K}$ for the temperature change during 100 years.

The number of breaks for US station appears extraordinary large. Menne et al. (2009), e.g., found only one significant break every 15 to 20 years. However, the latter estimate contains only the breaks which are large enough to be detected by a homogenization algorithm. Our approach, in contrast, comprises all breaks. It would imply that only one third of the breaks is usually detected. In Section 6 we show that such an assumption is not unrealistic. In contrast, the examination of a commonly used stop criterion, by which the number of detected breaks is determined, suggests actually a fraction smaller than 0.5 .

\section{Fraction of undetected breaks}

Detection algorithms search for the optimum number of breaks and their positions. For each number of breaks an optimum combination of positions exists, which is defined by explaining the maximum of variance. However, more breaks are of course able to explain more variance. Thus, to decide which number of breaks is optimal a stop criterion is needed that penalizes the insertion of breaks. Caussinus and Mestre (2004), Domonkos (2011) and Lindau and Venema (2018a) use the Lyazrhi stop criterion (Caussinus and Lyazrhi, 1997), which is given by:

$$
k_{0}=\min _{0 \leq k<n}\left(\ln (1-v)+\frac{2 k \ln (n)}{n-1}\right)
$$


$n$ denotes the length of the time series, $k$ the number of test breaks, and $v$ the fraction of variance explained by the segmentation. Thus, $k_{0}$, the number of detected breaks, can be obtained by setting the first derivative of Eq. (26) equal to zero:

$$
\frac{1}{1-v} \frac{d v}{d k}=\frac{2 \ln (n)}{n-1} \quad, \text { for } k=k_{0}
$$

The right-hand side of Eq. (27) is a constant, which is for monthly data (where $n \approx 1000$ ) equal to 0.014. Thus, Eq. (27) states that the search is stopped if an additional test break is not able to explain at least $1.4 \%$ of the so far unexplained variance.

The total (normalized) variance is equal to the sum of break variance and noise variance:

$$
v_{B}+v_{N}=1
$$

In the vicinity of the optimum solution $k_{0}$, most of the break variance is already explained and the unexplained variance will be approximately equal to the noise:

$$
1-v \cong v_{N} \text { for } k \cong k_{0}
$$

We substitute the unexplained variance in Eq. (27) by the above approximation and get:

$$
\frac{1}{v_{N}} \frac{d v}{d k}=\frac{2 \ln (n)}{n-1} \quad, \text { for } k=k_{0}
$$

For the ideal case without noise, Lindau and Venema (2018a) showed that the explained variance grows with $k$ by:

$$
v=v_{B}\left(1-\left(1-\frac{k}{n_{k}}\right)^{4}\right)
$$

where $n_{\mathrm{k}}$ denotes the true number of breaks. The derivation of Eq. (31) with respect to $k$ yields:

$$
\frac{d v}{d k}=\frac{4 v_{B}}{n_{k}}\left(1-\frac{k}{n_{k}}\right)^{3}
$$

If the method works perfectly it explains only break variance and no noise. Then we can insert Eq.

(32) into Eq. (30):

$$
\frac{4 v_{B}}{n_{k} v_{N}}\left(1-\frac{k_{0}}{n_{k}}\right)^{3}=\frac{2 \ln (n)}{n-1}
$$

Solved for the underestimation factor $f_{\mathrm{u}}$ :

$$
f_{u}=1-\frac{k_{0}}{n_{k}}=\sqrt[3]{\frac{\ln (n)}{2(n-1)} \frac{v_{N}}{v_{B}} n_{k}}
$$

To estimate $f_{\mathrm{u}}$, we insert the values found for RD breaks in the US given in Table 3. These are valid for monthly scale so that $n$ is in the order of 1000 (month). For such a time span 14 breaks (17.1 century $^{-1} \times 0.83$ century) are expected, which has to be doubled because two stations contribute their breaks in a difference time series: 


$$
f_{u}\left(n=1000, S N R=0.871, n_{k}=28\right)=0.503
$$

Thus, only about $50 \%$ of the breaks are detected, i.e. 14 of 28 , which means 7 per station.

For a Gaussian distribution $50.3 \%$ of the data lies inside 0.71 standard deviations. In Section 5 we found a standard deviation of $0.485 \mathrm{~K}$ for RD breaks. Thus, if the detectability is only a function of size, an estimate for the minimum detectable break size is:

$$
d=0.71 \times 0.48 \mathrm{~K}=0.344 \mathrm{~K}
$$

The above calculations provide of course only a rough estimate and for larger SNR (2) and less breaks ( 5 per station) the underestimation factor is considerably smaller:

$$
f_{u}\left(n=1000, S N R=2, n_{k}=10\right)=0.205
$$

Furthermore, we assumed a perfect performance of the search method. This means that it is solely the break variance which is gradually explained. However, Lindau and Venema (2018a) showed that in practice also some noise is erroneously treated as signal, which can lead, especially for SNRs far below 1 , to large errors. In the context discussed here, it is important that the detected number of breaks may increase by this effect. For SNRs of about 1 , as it is the case here, these errors are expected to remain limited.

Above, we estimated the detection threshold of US data to about $0.344 \mathrm{~K}$. An alternative way to calculate this parameter is the following. The potentially attainable (but just not accepted) increase of the explained variance at $k_{0}$ (for the last detected break) gives an estimate of the minimum detectable break variance. Using Eq. (30) we get:

$$
\Delta v=\frac{d v}{d k}\left(k_{0}\right)=\frac{2 \ln (n)}{n-1} v_{N}
$$

It is difficult to determine exactly which break size (jump height) corresponds to a specific amount of additionally explained variance. Any new solution for $k$ breaks will change all break positions of the previous solution for $k-1$ breaks. However, for a rough estimate we assume that one of the existing segments is divided in the middle, while the others are left unchanged, as it is applied in hierarchical splitting methods. In this case a jump height of $h$ will produce a variance of $h^{2} / 4$. However, as only one segment is affected, we have to multiply by its relative length. An estimate for that is $1 / k_{0}$ so that a rough estimate for the just not accepted variance gain is:

$$
\Delta v=\frac{h^{2}}{4 k_{0}}
$$

By combining Eq. (38) and (39) we can solve for the minimum break size $h$. Inserting $n=1000, k_{0}=14$ and $v_{\mathrm{N}}=0.155 \mathrm{~K}^{2}$ yields:

$$
h=\sqrt{8 k_{0} \frac{\ln (n)}{n-1} v_{N}}=\sqrt{8 \cdot 14 \cdot 0.0069 \cdot 0.155 \mathrm{~K}^{2}}=0.346 \mathrm{~K}
$$

This is in good agreement to the estimate of the detection threshold made in Eq. (36). 
The theoretical estimates above are rechecked by running the detection algorithm presented by Lindau and Venema (2018a). 1000 time series with the characteristics found for American RD breaks (length 1000 month, $\sigma_{\delta}^{2}=0.1175 \mathrm{~K}^{2}, \sigma_{\varepsilon}{ }^{2}=0.1550, n_{\mathrm{k}}=28$, see Eq. 35 ) are modelled as described in Section 3 and then analyzed by the search algorithm (Fig. 7). The histogram of the inserted jump heights is given by the crosses and follows the expected normal distribution $\mathrm{N}\left(0,2 \sigma_{\delta}{ }^{2}\right)$. The O-symbols denote the detected breaks. Large breaks are nearly entirely retrieved, whereas breaks sizes below $0.1 \mathrm{~K}$ are completely missing. The maxima are reached at the break size classes $\pm 0.5 \mathrm{~K}$, which is in accordance with Menne et al. (2009) and Dunn et al. (2014). In total 11,369 of 27,790 breaks are detected, which is equal to $40.9 \%$. This largely confirms our theoretical analysis (Eq. 35), which found $49.7 \%$.

\section{Discussion}

The above analysis found that the break signal of contiguous USA is a mixture of both Brownian Motion and Random Deviations, while in Germany it is dominated by Random Deviations. This fits qualitatively to the findings of the HOME benchmarking study, which found for several networks in Europe that the break signal was mostly Random Deviations, but also had a Brownian Motion component.

This gives us two independent lines of evidence that the break signal has both characteristics. As explained in Section 2 Venema et al. (2012) studied the sign of consecutive break pairs found by statistical homogenization. Thus Venema et al. (2012) analyse the break signal from one break to the next, while the method in this paper considers the characteristic of the break signal as whole. The current study furthermore, does not require a previous homogenization.

The SNR for the German observations is close to 1, which is twice as high as the ratio found by Lindau and Venema (2018a) for the German network. The smaller SNR in our older work may be caused by two reasons: The first is that in the earlier study only data from the second half of the $20^{\text {th }}$ century was considered where we expect smaller inhomogeneities. A second reason may be that previously neighbor stations with a maximum correlation were selected, whereas the present study uses the distance. Maximum correlation selects for both a similar climate, but also for a similar break signal. This reduces the break variance in the difference time series and consequently produces a smaller SNR. Thus, selecting station pairs with highest correlation may not be the best method to detect breaks.

The number of breaks detected by homogenization will be certainly lower than the actual number of breaks in a dataset. The main effect is that small breaks are not detected, but homogenization algorithms will also combine multiple subsequent breaks into one. Using the Pairwise Homogenization Algorithm (Menne and Williams, 2009), Dunn et al. (2014) found a break frequency of 6.8 breaks per century in the global dataset HadISD. For conterminous USA (USHCN) Menne et al. (2009) found a break frequency of 5 to 6.7 breaks per century. The minimum detectable break size is not well defined and hard to estimate from the histogram of detected breaks. This histogram is bimodal, it looks like a normal distribution where many values around zero are missing. If we take the two maxima of this histogram as a coarse estimate of the minimum detectable size, it is for both datasets about 1 standard deviation of the distribution of all breaks. That would mean that about 68 percent of all breaks are not detected. 
In Section 6 we analyze the behavior of a multiple breakpoint detection method, which largely confirms this estimate. It suggests that in case of the US data analyzed above we would only detect 40 to $50 \%$ of the actual break points. Homogenization detection algorithms combining subsequent breaks will further reduce this fraction so that one third is not unrealistic. Consequently, it seems reasonable that our method found much higher break frequencies than the previously reported significant break frequencies based on statistical homogenization.

For Germany our estimate of four breaks per century is comparable to Lindau and Venema (2018a), who found 6 breaks per century. As in this paper, they obtained the break rate directly from the data and without running a homogenization algorithm. The earlier estimate comprises 10 times more stations and only half of the time period, which could be the reason for such a difference. Four breaks per century correspond roughly to 1.3 to 2 breaks per century (one third to one half) that are large enough to be detected by a homogenization algorithm. This value is small, but in good agreement to the 1.5 breaks per century Müller-Westermeier (2003) found using SNHT for the long temperature series in the German network.

\section{Conclusions}

We presented a method to estimate the strength and frequency of inhomogeneities and to distinguish between Brownian Motion (BM) and Random Deviation (RD) type breaks. This is possible because the two break types produce different signals in the variance of spatiotemporal difference between two stations and between two dates. BM type breaks generate a linear increase of variance as a function of the time lag. The corresponding function for RD type breaks is saturating exponential; for noise it is a constant.

Applying the method to German data we found only a small number of stations (45 pairs), which fulfill the strict requirements concerning spatial closeness of the stations and temporal length and completeness. The results suggest that German stations contain only RD type breaks and no BM type breaks.

In the US much more data is available (1459 station pairs). The method clearly identifies both break types, BM and RD. RD breaks occur on average each 6 years and their size has a standard deviation of $0.34 \mathrm{~K}$. If expressed as jump height this value has to be multiplied by $\sqrt{2}$ so that $0.485 \mathrm{~K}$ results.

The number of breaks is, with 17 per century, three times larger than commonly estimated. However, our estimate includes all breaks, whereas the traditionally estimates are often denoted as significant breaks because they comprise only the detected ones. Thus, the total and the detected number of breaks are essentially two different parameters. As demonstrated in Section 6, there is some evidence from the theory that more than half of the breaks may be actually not detected during homogenization. Since they are of small size, their impact may be limited for the climatological practice. However, the new possibility to estimate also the total number of breaks could improve our insight into the homogenization problem.

If the inhomogeneities in benchmark datasets are modelled as BM type breaks they are probably easier detectable due to their large impact on the variance. Therefore, results from benchmark datasets with different break types are not directly comparable; not only strength and frequency of 
the breaks are crucial, but also the break type. A mixture of both breaks types seems reasonable at least if the conditions in the US shall be modelled.

\section{References}

Chimani B, Venema V, Lexer A, Andre K, Auer I, Nemec J. (2018) Inter-comparison of methods to homogenize daily relative humidity. Int. J. Climatol., 1-17. https://doi.org/10.1002/joc.5488.

Caussinus $\mathrm{H}$ and Lyazrhi F. (1997) Choosing a linear model with a random number of change-points and outliers. Ann. Inst. Stat. Math.49, 761-775.

Caussinus H and Mestre O. (2004) Detection and correction of artificial shifts in climate series, Appl. Statist., 53, 405-425.

Domonkos P. (2011) Adapted Caussinus-Mestre Algorithm for Networks of Temperature series (ACMANT), Int. J. Geosci., 2, 293-309.

Domonkos P. (2011) Efficiency evaluation for detecting inhomogeneities by objective homogenization methods, Theor. Appl. Climatol., 105, 455-467, doi:10.1007/s00704-011-0399-7.

Dunn RJH, Willett KM, Morice CP, and Parker DE. (2014) Pairwise homogeneity assessment of HadISD. Clim. Past, 10, 1501-1522, doi: 10.5194/cp-10-1501-2014.

Guijarro JA, López JA, Aguilar E, Domonkos P, Venema VKC, Sigró J, Brunet M. (2017) Comparison of homogenization packages applied to monthly series of temperature and precipitation. The MULTITEST project. $9^{\text {th }}$ Seminar for homogenization and quality control in climatological databases and $4^{\text {th }}$ conference on spatial interpolation techniques in climatology and meteorology (Budapest, April 3-7), WMO WCDMP-No.85, pp. 46-62.

Killick, RE. (2016) Benchmarking the Performance of Homogenisation Algorithms on Daily Temperature Data. PhD thesis, University of Exeter, Department of Mathematics, 249 pp.

Lindau, R. (2003) Errors of Atlantic Air-Sea Fluxes Derived from Ship Observations, Journal of Climate, 16 No.4, 783-788.

Lindau, R and Venema V. (2013) On the multiple breakpoint problem and the number of significant breaks in homogenization of climate records, Idöjaras - Quarterly Journal of the Hungarian Meteorological Service, 117, No. 1, 1-34.

Lindau R and Venema VKC. (2016) The uncertainty of break positions detected by homogenization algorithms in climate records. Int. J. Climatol., 36, No. 2, 576-589, doi: 10.1002/joc.4366.

Lindau R and Venema VKC. (2018a) The joint influence of break and noise variance on the break detection capability in time series homogenization. Adv. Stat. Clim. Meteorol. Oceanogr., 4, 1-18, https://doi.org/ 10.5194/ascmo-4-1-2018.

Lindau R and Venema VKC. (2018b) On the reduction of trend errors by the ANOVA joint correction scheme used in homogenization of climate station records. Int. J. Climatol., DOI: 10.1002/joc.5728. 
Menne MJ and Williams CN. (2009) Homogenization of Temperature Series via Pairwise Comparisons. J. Climate, 22, 1700-1717, https://doi.org/10.1175/2008JCLI2263.1

Menne MJ and Williams CN Jr. (2005) Detection of undocumented changepoints using multiple test statistics and composite reference series, J. Climate, 18, 4271-4286.

Menne MJ, Williams CN, and Vose RS. (2009) The U.S. Historical Climatology Network Monthly Temperature Data, Version 2. Bull. Amer. Meteor. Soc., 90, 993-1008.

https://doi.org/10.1175/2008BAMS2613.1

Müller-Westermeier G. (2003) Statistical analysis of results of homogeneity testing and homogenisation of long climatological time series in Germany. Fourth seminar for homogenization and quanlity control in climatological databases, Budapest, Hungary, 6-10 October 2003, report no. WCDMP-No. 56, WMO-TD No. 1236.

Rennie J, Lawrimore J, Gleason B, Thorne P, Morice C, Menne M, Williams C, Gambi de Almeida W, Christy J, Flannery M, Ishihara M, Kamiguchi K, Klein Tank A, Mhanda A, Lister D, Razuvaev V, Renom M, Rusticucci M, Tandy J, Worley S, Venema V, Angel W, Brunet M, Dattore B, Diamond H, Lazzara M, Le Blancq F, Luterbacher J, Mächel M, Revadekar J, Vose R, Yin X. (2014) The International Surface Temperature Initiative global land surface databank: monthly temperature data version 1 release description and methods. Geoscience Data Journal, 1, pp. 75-102, doi: 10.1002/gdj3.8

Venema V, Mestre O, Aguilar E, Auer I, Guijarno JA, Domonkos P, Vertacnik G, Szentimrey T, Stepanek P, Zahradnicek P, Viarre J, Muller-Westermeier G, Lakatos M, Williams CN, Menne MJ, Lindau R, Rasol D, Rustemeier E, Kalokythas K, Marinova T, Andresen L, Acquaotta F, Fratianni S, Cheval S, Klancar M, Brunetti M, Gruber C, Prohom Duran M, Likso T, Esteban P, and Brandsma T. (2012) Benchmarking homogenization algorithms for monthly data. Clim. Past, 8, 89-115.

Williams CN, Menne MJ, and Thorne PW. (2012) Benchmarking the performance of pairwise homogenization of surface temperatures in the United States. J. Geophys. Res., 117, D05116, doi: 10.1029/2011JD016761.

\section{Appendix: Internal and external variance of Brownian motion}

Consider a Brownian motion $y_{\mathrm{j}}$ with $k$ time steps that is given by:

$$
y_{j}=\sum_{i=1}^{j} x_{i} \quad, \quad 1 \leq j \leq k \quad, \quad x_{i} \sim N\left(0, \sigma_{\beta}^{2}\right)
$$

with $x_{i}$ being an independent normal distributed random variable. The expected value of the variance at a specific time step $j$ is:

$$
\operatorname{Var}\left(y_{j}\right)=\operatorname{Var}\left(\sum_{i=1}^{j} x_{i}\right)=j \sigma_{\beta}^{2}
$$

The total variance is equal to the mean over all time points: 


$$
\operatorname{Var}_{t o t}=\frac{1}{k} \sum_{j=1}^{k} j \sigma_{\beta}^{2}=\frac{\sigma_{\beta}^{2}}{k} \sum_{j=1}^{k} j=\frac{\sigma_{\beta}^{2}}{k} \frac{k(k+1)}{2}=\sigma_{\beta}^{2} \frac{k+1}{2}
$$

Thus, for large $k$, the total variance converges to:

$$
\operatorname{Var}_{\text {tot }}=\frac{k}{2} \sigma_{\beta}^{2}
$$

The external variance $\left(\operatorname{Var}_{\text {ext }}\right)$ is defined as the variance of the set of temporal means of the time series $(\bar{y})$. The mean of one time series is defined as:

$$
\bar{y}=\frac{1}{k} \sum_{j=1}^{k} y_{j}=\frac{1}{k} \sum_{j=1}^{k} \sum_{i=1}^{j} x_{i}=\frac{1}{k} \sum_{i=1}^{k}(k-i+1) x_{i}
$$

Since the expectation of the mean itself is zero, the external variance is equal to the squared mean $\bar{y} \bar{y}$ averaged over all time series, which is denoted by square brackets below. All mixed products between two different $x$ have the expectation zero, because they are independent and random. Only the squares themselves remain and they have the expectation $\sigma_{\beta}^{2}$. Thus, it results:

$$
\operatorname{Var}_{e x t}=[\bar{y} \bar{y}]=\frac{\sigma_{\beta}^{2}}{k^{2}} \sum_{i=1}^{k}(k-i+1)^{2}
$$

Setting in Eq. (A6) $m=k-i+1$ leads to:

$$
\operatorname{Var}_{\text {ext }}=\frac{\sigma_{\beta}^{2}}{k^{2}} \sum_{m=1}^{k} m^{2}=\frac{\sigma_{\beta}^{2}}{k^{2}} \frac{(2 k+1)(k+1) k}{6}=\frac{(2 k+1)(k+1)}{6 k} \sigma_{\beta}^{2}
$$

For large $k$ this converges to:

$$
\operatorname{Var}_{\text {ext }}=\frac{k}{3} \sigma_{\beta}^{2}
$$

A comparison of Eq. (A8) with Eq. (A4) shows that two thirds of the total variance is contributed by the external variance. The internal variance is equal to the remaining part, which is exactly given by subtracting Eq. (A7) from (A3):

$$
\operatorname{Var}_{\text {int }}=\sigma_{\beta}^{2}\left(\frac{k+1}{2}-\frac{(2 k+1)(k+1)}{6 k}\right)=\sigma_{\beta}^{2} \frac{k^{2}-1}{6 k}
$$

which converges for large $k$ to:

$$
\operatorname{Var}_{\text {int }}=\frac{k}{6} \sigma_{\beta}^{2}
$$

Thus, the internal variance is only a $1 / 3$ of the total variance. 
Table 1: 100 station pairs with 20 repetitions.

\begin{tabular}{|l|r|r|r|r|}
\hline \multicolumn{1}{|c|}{ Parameter } & \multicolumn{1}{|c|}{ Input } & Retrieved mean & Stand. Deviation & Relative error \% \\
\hline $\mathrm{b}$ in $10^{-2} \mathrm{~K}^{2} \mathrm{a}^{-1}$ & 1.000 & 0.987 & 0.317 & 32.1 \\
\hline $\mathrm{c} \mathrm{in} 10^{-2} \mathrm{a}^{-1}$ & 5.000 & 5.973 & 2.618 & 43.8 \\
\hline $\mathrm{d}$ in $\mathrm{K}^{2}$ & 0.400 & 0.421 & 0.184 & 43.7 \\
\hline e in $\mathrm{K}^{2}$ & 0.400 & 0.402 & 0.015 & 3.8 \\
\hline
\end{tabular}

Table 2: 1000 station pairs with 20 repetitions.

\begin{tabular}{|l|r|r|r|r|}
\hline \multicolumn{1}{|c|}{ Parameter } & \multicolumn{1}{|c|}{ Input } & Retrieved mean & Stand. Deviation & Relative error \% \\
\hline $\mathrm{b}$ in $10^{-2} \mathrm{~K}^{2} \mathrm{a}^{-1}$ & 1.000 & 1.040 & 0.129 & 12.4 \\
\hline $\mathrm{c}$ in $10^{-2} \mathrm{a}^{-1}$ & 5.000 & 5.519 & 1.112 & 20.1 \\
\hline $\mathrm{d}$ in $\mathrm{K}^{2}$ & 0.400 & 0.378 & 0.075 & 19.8 \\
\hline $\mathrm{e}$ in $\mathrm{K}^{2}$ & 0.400 & 0.398 & 0.007 & 1.6 \\
\hline
\end{tabular}

Table 3: Characteristic break parameters for US and German station data.

\begin{tabular}{|l|c|c|c|c|}
\hline & $\mathrm{p}_{\beta}\left(\sigma_{\beta}{ }^{2}\right)\left(\mathrm{K}^{2} \mathrm{a}^{-1}\right)$ & $\mathrm{p}_{\delta}{ }^{-1}$ (years) & $\sigma_{\delta}(\mathrm{K})$ & $\sigma_{\varepsilon}(\mathrm{K})$ \\
\hline US & 0.0045 & 5.8 & 0.34 & 0.39 \\
\hline Germany & 0.0000 & 24.4 & 0.36 & 0.34 \\
\hline
\end{tabular}




\section{Figures}

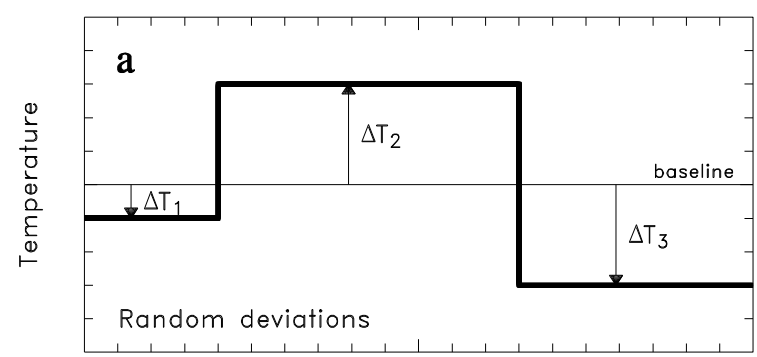

Time

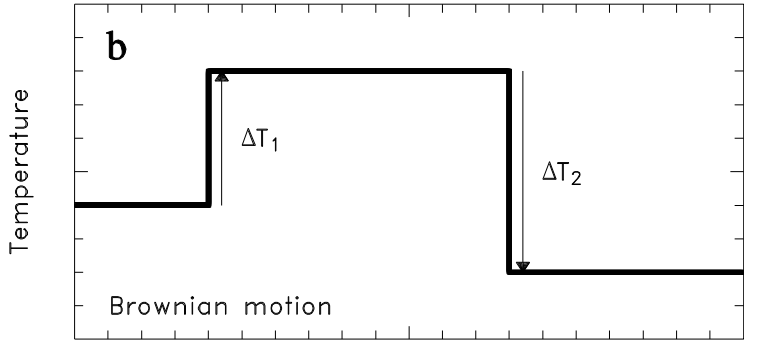

Time

Fig. 1: Conceptual model for the two kinds of breaks. For RD breaks (left panel) the deviation from a baseline is an independent variable, for BM (right panel) the jump heights are mutually independent.
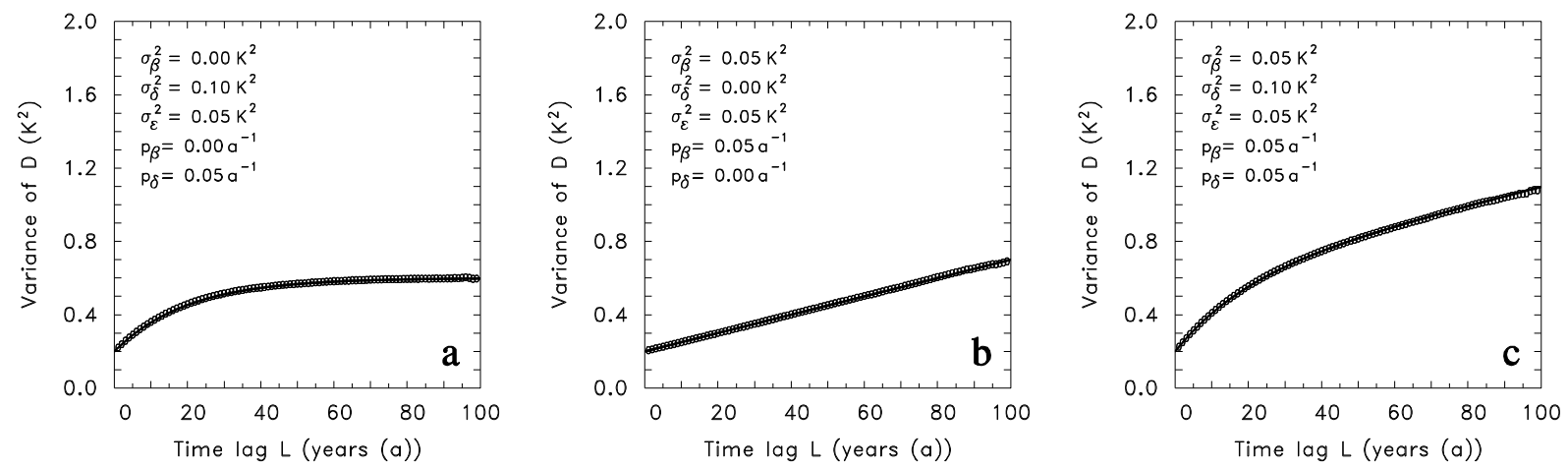

Fig. 2: Application of the method to simulated data using the input parameters given in the upper left corner. O-symbols denote the empirically obtained spatiotemporal variance based on 10,000 time series pairs. The expectation given by Eq. (20) is shown as fat line. In all three panels the noise variance $\sigma_{\varepsilon}{ }^{2}$ is equal to $0.05 \mathrm{~K}^{2}$. Panel a) shows RD type breaks alone, b) BM type breaks alone c) both breaks types combined. 

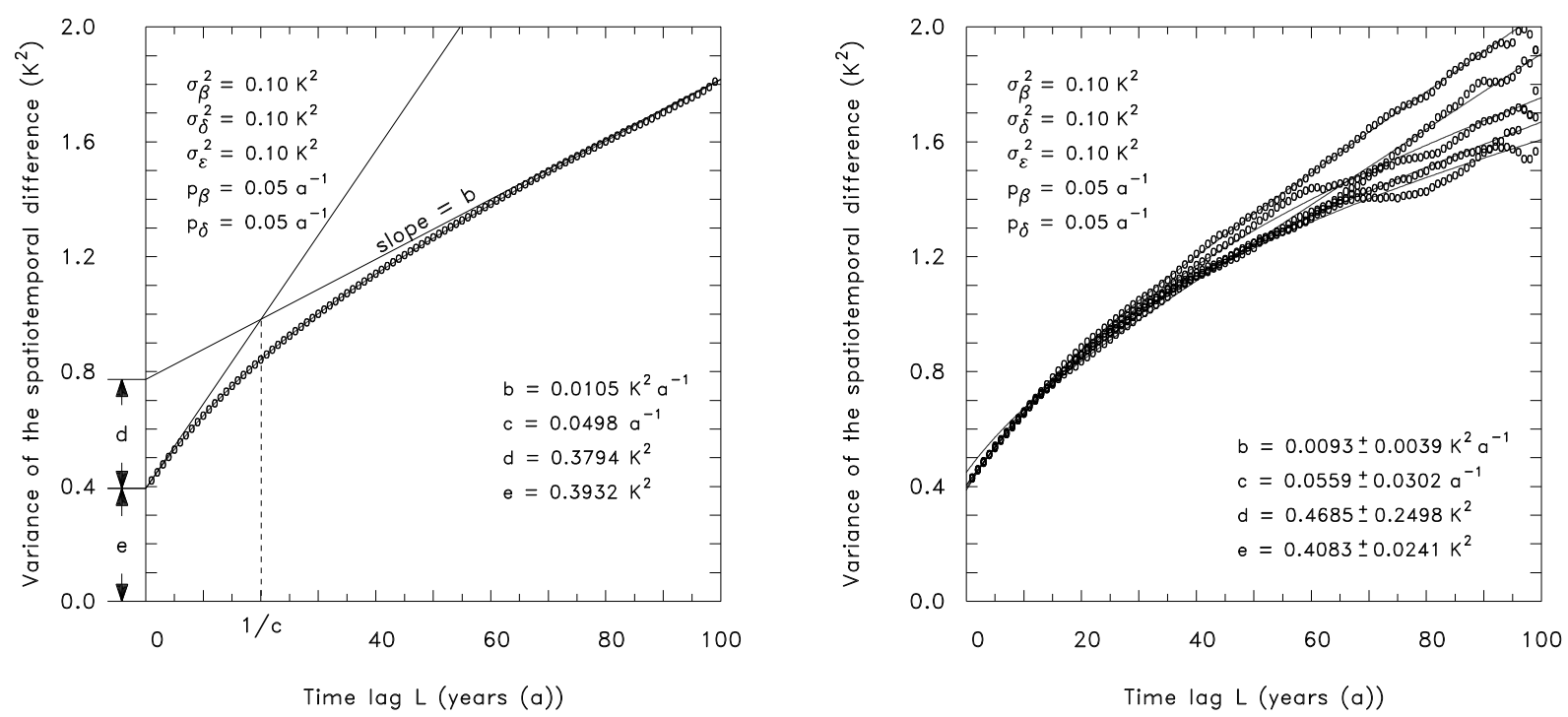

Fig. 3: Illustration for the retrieval of the four characteristic parameters b, c, d, and e, which are numerically given in the lower right corner. 10,000 time series pairs are generated using the input parameters given in the upper left corner. The empirical data is given by O-symbols. A function of the form of Eq. (21) is fitted, which is given by the closely following continuous curve. The two tangents, separately fitted for small and large lags, can be used for a geometrical interpretation of the retrieved parameters.

Fig. 4: As Fig. 3, but for a data volume reduced from 10,000 to 100 pairs. The variability of five random repetitions illustrates the increased uncertainty.
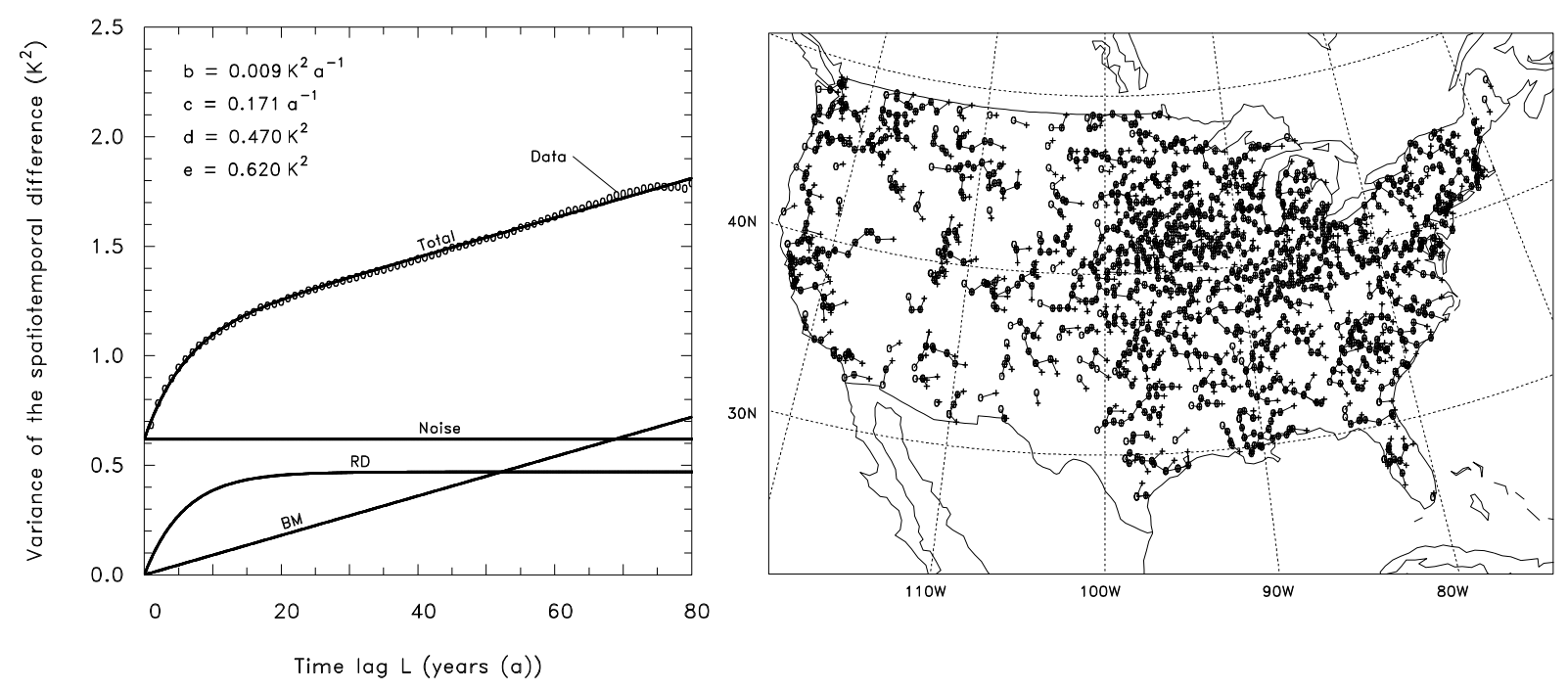

Fig. 5: Variance of the spatiotemporal difference for US station pairs (left panel). The obtained data for each time lag class is given by $\mathrm{O}$-symbols. The fitted function according to Eq. (21) is given by the upper continuous curve that closely follows the data. Additionally, the three individual variance signals for BM breaks, RD breaks, and noise are given. The right panel shows the distribution of the 1459 station pairs used. 

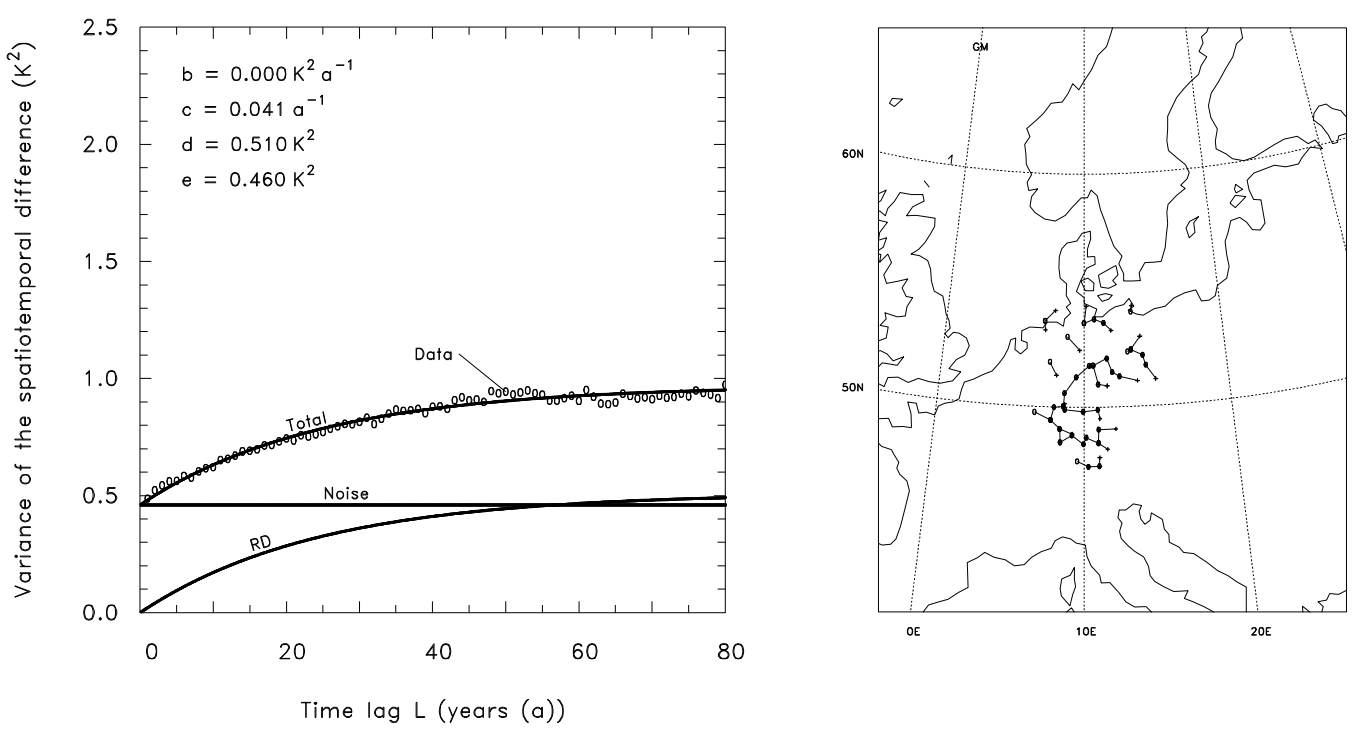

Fig. 6: As Figure 5, but for 45 station pairs in Germany.

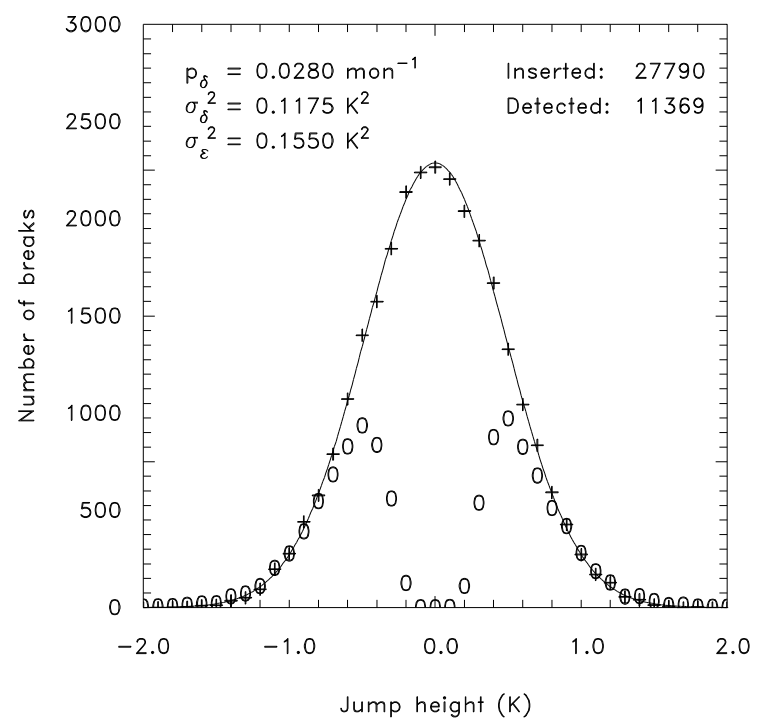

Fig. 7: Histogram for the number of inserted $(+)$ and detected $(0)$ breaks as found by the search algorithm of Lindau and Venema (2018a). 1000 time series with the statistical properties of RD breaks in US stations are modelled and tested. The curve gives the expected distribution of the inserted break sizes. 